\title{
Research, Plastic Surgery, and Archives of Plastic Surgery
}

Won Jai Lee

Co-editor, Archives of Plastic Surgery

Department of Plastic and Reconstructive Surgery, Yonsei University College of Medicine, Seoul, Korea

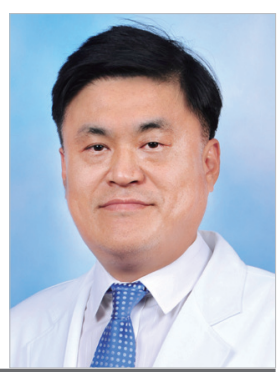

A medical doctor is a professional whose job is to treat people who are sick or hurt. Not only are plastic surgeons medical doctors, but surgeons who have the special skill of performing operations. Using our knowledge and techniques, we can provide good functionality and aesthetically satisfactory results to patients. Surgeons have the tremendous privilege of working in the operative field. This is a truly worthy endeavor that requires considerable experience and continuous learning. Within medicine, plastic surgery is a highly clinical field, so many plastic surgeons have focused their efforts on improving clinical results, and particularly on improving surgical techniques. Of course, the surgical skill or technique of a plastic surgeon is directly associated with the surgical results. Clearly, good surgical decisionmaking and surgical skills are of paramount importance for being a good plastic surgeon. However, is this enough?

Sometimes, we feel that an improved technique, new idea, or novel concept that we developed should be validated and disseminated among other plastic surgeons. At that point, carefully chosen experimental methods can confirm the validity of our innovation. All these activities are included in research. Research is never far from the entire scope of our clinical activities. Moreover, research-based treatment can provide a solid longterm base for the progress of plastic surgery. We are all eager to improve our field, so that future patients can be treated with better treatment options. This can be accomplished by research, because plastic surgery is a medical science, not a series of anecdotal experiences.

Research falls into certain categories, including basic research, preclinical research, translational research, and clinical research. Basic research usually refers to experimental studies at the cell and molecular levels. Basic research is commonly conducted by $\mathrm{PhD}$ scientists, although some medical doctors successfully pursue basic research. Preclinical research refers to studies conducted to test a drug, a procedure, or another medical treatment in animals. Preclinical studies are performed to collect data in support of the safety and efficacy of a new investigational treatment. Preclinical studies are required before clinical trials in humans can be started [1]. Clinical research refers to studies conducted among patients. It can be prospective or retrospective. Certain surgical skills and treatment strategies can be validated by obtaining relevant evidence through clinical studies.

Archives of Plastic Surgery (APS) receives a huge quantity of manuscripts describing plastic surgery research. I want to express gratitude for all the authors and their dedication to their research. However, most of the submitted manuscripts are clinical studies. I think this is natural, because plastic surgery is a clinically oriented field of medicine. Nonetheless, we face a new era in our field. Government investment into biological research is increasing every year throughout the world [2]. One of the most advanced frontiers of modern medicine, regenerative medicine, is closely related to plastic surgery. As global economic conditions improve, quality of life becomes more important, and the role of the plastic surgeon is therefore increasing in scope and importance.

It is time to pay more attention to research beyond clinical studies. An analysis of the articles published in APS revealed a numerical imbalance between clinical research and non-clinical research. We look forward to receiving more non-clinical-that is, experimental, translational, and preclinical—studies as submissions. 


\section{REFERENCES}

1. "Medical research". Wikipedia [Internet]. Wikimedia Foundation Inc.; [cited 2017 Aug 30]. Available from: https:// en.wikipedia.org/wiki/Medical_research.

2. Chakma J, Sun GH, Steinberg JD, et al. Asia's ascent: global trends in biomedical R\&D expenditures. N Engl J Med 2014;370:3-6.
Correspondence: Won Jai Lee

Department of Plastic and Reconstructive Surgery, Yonsei University College of Medicine, 50-1

Yonsei-ro, Seodaemun-gu, Seoul 03722, Korea

Tel: +82-2-2228-2210, Fax: +82-2-361-6947, E-mail: pswjlee@yuhs.ac

No potential conflict of interest relevant to this article was reported.

Received: 21 Aug 2017 • Revised: 28 Aug 2017 • Accepted: 30 Aug 2017

pISSN: 2234-6163・ elSSN: 2234-6171

https://doi.org/10.5999/aps.2017.44.5.359 • Arch Plast Surg 2017;44:359-360 